\title{
General perioperative management of gastric cancer patients at high-volume centers
}

\author{
Hye Seong Ahn · Jeong Hwan Yook - Cho Hyun Park • Young Kyu Park • Wansik Yu • \\ Moon-Soo Lee $\cdot$ Han Sang-Uk $\cdot$ Keun Won Ryu $\cdot$ Tae Sung Sohn $\cdot$ Hyung-Ho Kim • \\ Seung Ho Choi $\cdot$ Sung Hoon Noh $\cdot$ Naoki Hiki $\cdot$ Takeshi Sano $\cdot$ Han-Kwang Yang
}

Received: 6 April 2010/Accepted: 31 October 2010/Published online: 5 March 2011

(c) The International Gastric Cancer Association and The Japanese Gastric Cancer Association 2011

\begin{abstract}
Background Gastric cancer is very common in Korea and Japan, where many hospitals annually perform high numbers of gastrectomies for gastric cancer. The aim of this study was to compare the general management of gastric cancer in high-volume centers in Korea and Japan.
\end{abstract}

\footnotetext{
H. S. Ahn · H.-K. Yang $(\square)$

Department of Surgery and Cancer Research Institute,

Seoul National University College of Medicine,

28 Yeongeon-dong, Jongno-gu, Seoul 110-744, Korea

e-mail: hkyang@snu.ac.kr

J. H. Yook

Department of Surgery, Asan Medical Center, Seoul, Korea

C. H. Park

Department of Surgery, Catholic University Seoul St. Mary

Hospital, Seoul, Korea
}

\section{Y. K. Park}

Department of Surgery, Chonnam National University Hospital,

Gwangju, Korea

W. Yu

Department of Surgery, Kyungpook National University

Hospital, Daegu, Korea

\section{M.-S. Lee}

Department of Surgery, Soon Chun Hyang University Cheonan Hospital, Cheonan, Korea

H. Sang-Uk

Department of Surgery, Ajou University Hospital, Suwon, Korea

K. W. Ryu

Division of Gastric Cancer, National Cancer Center,

Goyang, Korea
Methods We undertook a survey of the general management of gastric cancer at high-volume centers (over 200 cases/year) and analyzed the answers.

Results In six of 14 hospitals surveyed, antimicrobial prophylaxis for elective gastrectomy was administered until postoperative day 3. A Levin tube and an abdominal drain were routinely inserted in seven and ten hospitals, respectively. Laboratory tests, such as complete blood cell

\section{T. S. Sohn}

Department of Surgery, Samsung Medical Center,

Sungkyunkwan University School of Medicine, Seoul, Korea

\section{H.-H. Kim}

Department of Surgery, Seoul National University Bundang Hospital, Seongnam, Korea

\section{S. H. Choi}

Department of Surgery, Yonsei University Kangnam Severance Hospital, Seoul, Korea

\section{S. H. Noh}

Department of Surgery, Yonsei University Severance Hospital, Seoul, Korea

N. Hiki

Cancer Institute Hospital of Japanese Foundation for Cancer Research, Tokyo, Japan

T. Sano

National Cancer Center, Tokyo, Japan

Present Address:

T. Sano

Cancer Institute Hospital of Japanese Foundation for Cancer

Research, Tokyo, Japan 
count, liver function test, electrolytes, and blood urea nitrogen/creatinine were performed frequently on postoperative days $1,2,3$, and 5 . Sips of water after open distal gastrectomy were restarted up to postoperative day 3 in twelve hospitals. The surgical pathology was reported up to postoperative day 10 in thirteen hospitals. Twelve hospitals provided a regular patient education program and only one hospital provided an integrated education program which included the participation of a surgeon, an oncologist, a nurse, and a nutritionist.

Conclusions The general management of gastric cancer in 14 high-volume centers was not so different among the centers. The general management protocols noted here are expected to provide useful information for perioperative care.

Keywords Gastric cancer · General management .

High-volume center

\section{Introduction}

Gastric cancer is the one of the most serious health problems in Korea and Japan [1, 2]. However, the overall survival rates after surgical treatment have been increasing and the rate of postoperative complications has decreased $[2,3]$. Probably, these improvements are due to the introduction of more radical surgical techniques ; early detection; and the improvement of anesthesia, perioperative care, and nutritional support. In addition, because of the high incidence of gastric cancer, many surgeons in Korea and Japan have accumulated substantial experience in the management of the disease, and have developed general protocols of perioperative management for patients with gastric cancer. However, there are several controversial issues in the general perioperative management of gastric cancer, such as Levin tube decompression, abdominal drain insertion, and antimicrobial prophylaxis. The aims of this study were to compare the general perioperative management of patients with gastric cancer in Korean and Japanese high-volume centers and to contribute to the improvement of surgical outcomes and to the development of site-specific protocols.

\section{Methods}

A brief questionnaire about the general perioperative management of patients with gastric cancer was sent in 2007, via email, to representative surgeons at Korean and Japanese high-volume centers where more than 200 operations were performed per year. In addition, two leading cancer centers in Japan were invited to participate in the surveillance. The survey form included 12 items, shown as Table 1. Each item was analyzed and compared among the institutions. For descriptive analysis, SPSS version 12.0 (SPSS, Chicago, IL, USA) was used.

Table 1 Form used for the survey of general perioperative management implemented at each high-volume center

(1) If different pathways are applied on a case-by-case basis (i.e., early gastric cancer vs. advanced gastric cancer; subtotal gastrectomy vs. total gastrectomy), please duplicate the relevant question box and fill out the answers separately

(2) If some items are difficult to assess, leave the corresponding box empty

\begin{tabular}{ll}
\hline Item & Answer \\
\hline Where is preoperative workup performed? & (1) At outpatient clinic (OPD) \\
(2) after admission & Regimen: \\
Prophylactic antibiotics & Duration: \\
Analgesics & Regimen: \\
Levin tube & Duration: \\
& (1) Inserted (2) not inserted \\
Abdominal drainage tube & Duration: \\
For subtotal and total gastrectomy & (1) Inserted (2) not inserted \\
If different, please describe separately & Duration: \\
Postoperative laboratory tests & What items? \\
Main type of fluid infused after surgery & When? \\
Schedule for oral intake & \\
Schedule for discharge & \\
Time required to receive pathology report (days) & \\
EMR (electronic medical record) -based critical pathway (CP) & (1) Performed (2) not performed \\
Regular patient education program & Interval of education: \\
& Education time: \\
\hline
\end{tabular}




\section{Results}

The answers from representative surgeons at 14 hospitals were analyzed.

Preoperative workup was performed after admission in 3 hospitals, and most workup was done before admission in 11 hospitals.

In three hospitals (3/14), antimicrobial prophylaxis for elective gastrectomy was administered for $24 \mathrm{~h}$ or less; it was administered up to postoperative day 2 in five hospitals and up to postoperative day 3 in six. The most frequently administered antibiotic was a second-generation cephalosporin $(n=8)$, followed by a first-generation cephalosporin $(n=4)$ and a third-generation cephalosporin $(n=2)$. In four hospitals, a cephalosporin and an aminoglycoside were chosen for prophlyaxis.

For pain control, patients received patient-controlled anesthesia (PCA) intravenously $(n=9)$ or epidurally $(n=5)$ until postoperative day 2 or 3 .

Surgeons at five hospitals $(5 / 14)$ did not insert a Levin tube preoperatively. However, in seven hospitals (7/14), the tube was inserted preoperatively and removed on postoperative day $1(n=5)$ or just after surgery $(n=2)$. Surgeons at another two hospitals (2/14) inserted a tube only in the patients with advanced gastric cancer or obstruction.

Surgeons at two (2/14) hospitals did not insert an abdominal drain, and surgeons at two other hospitals inserted abdominal drains on a case-by-case basis. However, surgeons at ten (10/14) hospitals inserted one or two abdominal drains according to the extent of resection (total or distal gastrectomy) or according to the anastomosis method (Billroth I or Billroth II). Although the time of drain removal varied from postoperative day 2 to postoperative day 6 , the most frequent postoperative days of drain removal were postoperative days $3-4(n=7)$.

Samples for laboratory tests were taken more than once. Usually the tests included a complete blood cell count, liver function test, electrolytes, blood urea nitrogen/creatinine, C-reactive protein, and chest X-ray. The most frequent time for laboratory tests was postoperative day 1 $(n=8)$ followed by postoperative days $2(n=6), 5$ $(n=5)$, and $3(n=4)$.

Five or ten percent dextrose solutions were infused in six hospitals prior to the initiation of oral diet. In four hospitals, patients received peripheral total parenteral nutrition. The status of use of other fluids such as amino acids or lipids could not be compared due to the limited number of answers.

Sips of water after open distal gastrectomy were restarted on postoperative day $3(n=9)$ and on postoperative day $2(n=3)$. Surgeons at some hospitals answered that the day of oral diet resumption was different according to the surgical approach (open or laparoscopic) and the extent of resection. In 2 hospitals, patients who underwent total gastrectomy resumed oral diet later than patients who underwent subtotal gastrectomy. And surgeons at three hospitals answered that patients who underwent laparoscopic gastrectomy resumed oral diet earlier than patients who underwent open gastrectomy.

Patients who underwent subtotal gastrectomy were discharged on postoperative day 6 or $7(n=7), 8$ or 9 $(n=4), 10$ or $11(n=2)$, or $5(n=1)$. At three hospitals, patients who underwent total gastrectomy were discharged 1-3 days later than patients who underwent subtotal gastrectomy. And surgeons at two hospitals answered that patients who underwent laparoscopy-assisted gastrectomy were discharged 1 or 2 days earlier than patients who underwent open gastrectomy. Patients in two Japanese hospitals seemed to stay longer than patients in Korean hospitals (10.5-11 vs. 6-9 days).

Surgical pathology was reported until postoperative day 10 at thirteen hospitals. In 3 hospitals only 3-5 days after surgery was required to get these reports. Two Japanese hospitals seemed to require more time (up to 28 days) than the Korean hospitals, especially when the tumor metastasis to lymph nodes.

Six hospitals had electronic medical record-based critical pathways and the other hospitals had a printed order set. Twelve hospitals provided a regular patient education program and nine hospitals had a nutritional counseling and support system. Four hospitals had group-educational programs and the other ten hospitals seemed to have only individual programs begun when the patient restarted oral diet or was discharged. Only one hospital provided an integrated education program which included the participation of a surgeon, a medical oncologist, a nurse, and a nutritionist.

\section{Discussion}

The mortality and morbidity after surgery for many cancers other than gastric cancer are known to be influenced by the hospital patient volume $[4,5]$. The existence of many highvolume centers for gastric cancer in Korea and Japan may have led to the low morbidity and mortality observed for patients after radical gastrectomy with lymphadenectomy $[6,7]$.

As for antimicrobial prophylaxis, according to the Centers for Disease Control (CDC) guidelines and references, a first-generation cephalosporin should be administered as antimicrobial prophylaxis in clean-contaminated operations within $30 \mathrm{~min}$ of the first surgical incision, with intraoperative supplemental administration every $3-4 \mathrm{~h}$ and postoperative administration for $24 \mathrm{~h}$ or less [8]. The 
Health Insurance Review and Assessment Service of Korea has recommended that a first- or second-generation cephalosporin should be administered as prophylaxis in gastrointestinal surgery, and administration should be started within $30 \mathrm{~min}$ or $1 \mathrm{~h}$ of skin incision and last for $24 \mathrm{~h}$ or less; in addition they note that aminoglycosides are not suitable for prophylaxis because of renal toxicity and ototoxicity [9]. Although the present study showed many discrepancies between guidelines and actual practice, the risk and benefit should be balanced for the appropriate use of antimicrobial prophylaxis.

Recently several studies, albeit with limited scientific evidence levels, have reported that Levin tube decompression was not correlated with earlier recovery of bowel function, shorter hospital stay, reduced anastomotic leakage, or fewer pulmonary complications after gastrectomy for gastric cancer [10, 11]. These findings might explain why surgeons in 5 of the 14 hospitals did not insert a Levin tube. With regard to abdominal drain tubes, prophylactic drain placement has been widely practiced by gastric surgeons. Surgeons who inserted abdominal drains might believe that the prophylactic use of drains provides early information about such factors as anastomotic leakage and intraabdominal bleeding. However, these benefit of prophylactic use of drain was not proven in two studies [12, 13]. The placement of a Levin tube and an abdominal drain in operations for gastric cancer warrants further investigation through large-scale randomized clinical trials.

Although many studies have shown that early oral feeding is feasible after gastrectomy, the optimal dietary schedule has not been established [14, 15]. Traditionally, postoperative oral intake after abdominal surgery was slowly and carefully introduced, due to anstomotic leakage and postoperative paralytic ileus. Malnutrition as one symptom arising from gastric cancer or one major complication after radical gastrectomy is known to be related to the quality of life, morbidity and mortality, and survival of patients after gastrectomy [16, 17]. These factors seemed to lead many hospitals in this study to adopt a policy of early oral intake and to implement nutritional counseling programs or group-educational programs.

There was a tendency in the present study that patients who underwent laparoscopic surgery resumed oral feeding earlier and were discharged later than patients who underwent open gastrectomy, although not all participants answered that there were different protocols for patients who underwent laparoscopic gastrectomy. Although two Japanese hospitals could not be taken to represent all hospitals in Japan, patients in these two hospitals were discharged relatively later than patients in the Korean hospitals, and the surgical pathology reports in the Japanese hospitals required more time, too. This longer hospital stay is in accordance with many reports showing a mean postoperative hospital stay of 15-32 days in Japan, which is relatively much longer than that in Korean hospitals (7-13 days) [6, 18-21]. The longer hospital stay in the Japanese institutions might reflect differences in the medical insurance systems.

\section{Conclusion}

The general perioperative management of gastric cancer patients at 14 high-volume centers was not so different among the hospitals, except that the hospital stay and the time required for obtaining surgical pathology reports were relatively longer in the Japanese hospitals than in the Korean hospitals. The general perioperative management information obtained in the present study could help many gastric surgeons to establish their own protocols and to improve surgical outcomes.

\section{References}

1. Lee HJ, Yang HK, Ahn YO. Gastric cancer in Korea. Gastric Cancer. 2002;5(3):177-82.

2. Maehara Y, Kakeji Y, Oda S, Takahashi I, Akazawa K, Sugimachi K. Time trends of surgical treatment and the prognosis for Japanese patients with gastric cancer. Br J Cancer. 2000;83(8): 986-91.

3. Borch K, Jonsson B, Tarpila E, Franzen T, Berglund J, Kullman $\mathrm{E}$, et al. Changing pattern of histological type, location, stage and outcome of surgical treatment of gastric carcinoma. Br J Surg. 2000;87(5):618-26.

4. Begg CB, Cramer LD, Hoskins WJ, Brennan MF. Impact of hospital volume on operative mortality for major cancer surgery. JAMA. 1998;280(20):1747-51.

5. Schrag D, Cramer LD, Bach PB, Cohen AM, Warren JL, Begg $\mathrm{CB}$. Influence of hospital procedure volume on outcomes following surgery for colon cancer. JAMA. 2000;284(23):3028-35.

6. Park DJ, Lee HJ, Kim HH, Yang HK, Lee KU, Choe KJ. Predictors of operative morbidity and mortality in gastric cancer surgery. Br J Surg. 2005;92(9):1099-102.

7. Kodera Y, Sasako M, Yamamoto S, Sano T, Nashimoto A, Kurita A. Identification of risk factors for the development of complications following extended and superextended lymphadenectomies for gastric cancer. Br J Surg. 2005;92(9):1103-9.

8. Mangram AJ, Horan TC, Pearson ML, Silver LC, Jarvis WR. Guideline for Prevention of Surgical Site Infection, 1999. Centers for Disease Control and Prevention (CDC) Hospital Infection Control Practices Advisory Committee. Am J Infect Control. 1999;27(2):97-132.

9. http://www.hira.or.kr/common/dummy.jsp?pgmid=HIRAB03020 2010000 Accessed 20 Oct 2010.

10. Doglietto GB, Papa V, Tortorelli AP, Bossola M, Covino M, Pacelli F. Nasojejunal tube placement after total gastrectomy: a multicenter prospective randomized trial. Arch Surg 2004;139(12):1309-13; discussion 13.

11. Yang Z, Zheng Q, Wang Z. Meta-analysis of the need for nasogastric or nasojejunal decompression after gastrectomy for gastric cancer. Br J Surg. 2008;95(7):809-16. 
12. Kim J, Lee J, Hyung WJ, Cheong JH, Chen J, Choi SH, et al. Gastric cancer surgery without drains: a prospective randomized trial. J Gastrointest Surg. 2004;8(6):727-32.

13. Kumar M, Yang SB, Jaiswal VK, Shah JN, Shreshtha M, Gongal R. Is prophylactic placement of drains necessary after subtotal gastrectomy? World J Gastroenterol. 2007;13(27):3738-41.

14. Heslin MJ, Latkany L, Leung D, Brooks AD, Hochwald SN, Pisters PW, et al. A prospective, randomized trial of early enteral feeding after resection of upper gastrointestinal malignancy. Ann Surg 1997;226(4):567-77; discussion 77-80.

15. Hirao M, Tsujinaka T, Takeno A, Fujitani K, Kurata M. Patientcontrolled dietary schedule improves clinical outcome after gastrectomy for gastric cancer. World J Surg. 2005;29(7):853-7.

16. Bae JM, Park JW, Yang HK, Kim JP. Nutritional status of gastric cancer patients after total gastrectomy. World J Surg 1998; 22(3):254-60; discussion 60-1.

17. Sategna-Guidetti C, Bianco L. Malnutrition and malabsorption after total gastrectomy. A pathophysiologic approach. J Clin Gastroenterol. 1989;11(5):518-24.
18. Kitano S, Shiraishi N, Kakisako K, Yasuda K, Inomata M, Adachi Y. Laparoscopy-assisted Billroth-I gastrectomy (LADG) for cancer: our 10 years' experience. Surg Laparosc Endosc Percutan Tech. 2002;12(3):204-7.

19. Ishikawa M, Kitayama J, Kaizaki S, Nakayama H, Ishigami H, Fujii S, et al. Prospective randomized trial comparing Billroth I and Roux-en-Y procedures after distal gastrectomy for gastric carcinoma. World J Surg 2005;29(11):1415-20; discussion 21.

20. Adachi Y, Shiraishi N, Shiromizu A, Bandoh T, Aramaki M, Kitano S. Laparoscopy-assisted Billroth I gastrectomy compared with conventional open gastrectomy. Arch Surg. 2000; 135(7):806-10.

21. Kim MC, Kim W, Kim HH, Ryu SW, Ryu SY, Song KY, et al. Risk factors associated with complication following laparoscopyassisted gastrectomy for gastric cancer: a large-scale Korean multicenter study. Ann Surg Oncol. 2008;15(10):2692-700. 